\title{
Destruction of Peierls dimerization in the molecular crystal model: Effects of quantum phonon fluctuations
}

\author{
C.Q. Wu, ${ }^{*}$ Q.F. Huang, and X. Sun \\ T.D. Lee Physics Laboratory and Department of Physics, \\ Fudan University, Shanghai 200433, China
}

(Received 2 July 1995)

\begin{abstract}
Effects of quantum phonon fluctuations on the Peierls dimerization in the one-dimensional molecular crystal model are reexamined by a functional integral approach. An equation for the dimerization order parameter is obtained within a one-loop approximation. The destruction of Peierls dimerization is found by taking the quantum phonon fluctuations into account for the spin- $1 / 2$ electron system. The consistency and discrepancy of the result with previous ones are discussed.
\end{abstract}

PACS numbers: 63.20.Kr, 05.40.+j, 64.90.+b Ms code: BGR576

In a one-dimensional electron-phonon system, the metallic phase is unstable against the electron-phønon interaction, which results in the Peierls transition. 1 At the half-filling case, a dimerized lattce will be formed and the ground state is of a dimerized long-range order. This is true for an arbitrarily small electronphonon coupling within the mean-field adiabatic approximation which treats the phonon degree of freedom classically. It is an interesting problem whether the dimerized ground state survives the quantum phonon fluctuations. There have been many works to study this problem. Among them are the renormalization group analysis plus the Monte Carlo numerical falculations, 3 on the Su-Schrieffer-Heeger $(\mathrm{SSH})$ modelt and the mplecular crystal (MC) model, the variational approach 60 on the same models, and the one-loop approximation on the SSH model 8 and the halogen- briqfed mixed-valence linear chain complexes (MX) model.

In the SSH model as well as the MX model, the optical phonon-electron interactions are dominant and the acoustic phonon-electron interactions are weak, so the quantum fluctuations are mainly induced by the optical phonon-electron couplings. It has been shown that the dimerization of lattice survives the quantum phonon fluctuations for the spin- $\frac{1}{2}$ systems whereas it is broken for the spinless systems. 19 However, in the MC model, the interactions between electrons and two branches of phonons are equally important, it seems that the quantum fluctuations should be much stronger than that in the SSH and MX models. Although the renormalizationgroup analysis shows absence of dimerized long range order for the zero mass system, Hirsch and Fradkin concluded that the ground state is dimerized for any nonzero mass systems from the analysis of symmetries as well as their Monte Carlo numerical simulations.

In this paper, we reexamine the effects of quantum phonon fluctuations on Peierls dimerizaton in the molecular-crystal model by a functional integral approach. An equation for the dimerization order parameter is obtained within a one-loop approximation. We find the Peierls dimerization will be destroyed by tak- ing the quantum phonon fluctuations into account for the spin- $\frac{1}{2}$ electron system. An equivalency between the spin- $\frac{1}{2}$ MC model and the spinless SSH or MX model is shown. The consistency and discrepancy of our results with the renormalization- group analysis as well as the Monte Carlo numerical simulations are discussed.

The one-dimensional molecular-crystal madel we consider in this paper takes the following form,

$$
\begin{aligned}
H= & \sum_{l}\left[\frac{1}{2 M} p_{l}^{2}+\frac{1}{2} K u_{l}^{2}\right] \\
& -\sum_{l, s} t_{0}\left(c_{l, s}^{\dagger} c_{l+1, s}+c_{l+1, s}^{\dagger} c_{l, s}\right)+\sum_{l, s} \alpha u_{l} c_{l, s}^{\dagger} c_{l, s} .
\end{aligned}
$$

where $c_{l, s}^{\dagger}$ and $c_{l, s}$ are the creation and annihilation operators of electrons sitting at molecular $l$ with spin $s, u_{l}$ (its conjugated momentum is $p_{l}$ ) describes an internal degree of freedom of molecule $l, t_{0}, \alpha$ and $K$ are the constants for the electron hopping, electron-phonon coupling and lattice elasticity, and $M$ is the mass of the internal vibrations in molecules which form the one-dimensional lattice. The model can also be viewed as describing the coupling between electrons and excitons with boson characters.

To take into account the quantum phonon fluctuations, we employ a functional integral approach. The partition function of the system can be written as a functional integral over both complex and Grassmann variables:

$$
Z=\int \mathcal{D}[u] \mathcal{D}\left[\phi^{*} \phi\right] e^{-S},
$$

where the action $S$ is

$$
\begin{aligned}
S & =\int_{0}^{\beta} d \tau\left\{\sum_{l}\left[\frac{1}{2} K u_{l}^{2}+\frac{1}{2} M\left(\partial u_{l} / \partial \tau\right)^{2}\right]\right. \\
& +\sum_{l, s} \phi_{l, s}^{*}\left(\partial_{\tau}-\mu\right) \phi_{l, s} \\
& -\sum_{l, s} t_{0}\left(\phi_{l, s}^{*} \phi_{l+1, s}+\phi_{l+1, s}^{*} \phi_{l, s}\right)
\end{aligned}
$$




$$
\left.+\sum_{l, s} \alpha u_{l} \phi_{l, s}^{*} \phi_{l, s}\right\}
$$

By making Fourier transformations on the integral variables

$$
u_{l}(\tau)=\frac{1}{\sqrt{\beta L}} \sum_{q, \Omega} e^{-i(q l+\Omega \tau)} u_{q}(\Omega),
$$

and similarly for $\phi_{l, s}^{*}$ and $\phi_{l, s}$, then for the phonon variables we have two branches on a dimerized lattice, that is, the acoustic phonon $a_{q}(\Omega)\left[\equiv u_{q}(\Omega)\right]$ and the optical phonon $b_{q}(\Omega)\left[\equiv u_{q+Q}(\Omega)\right], Q=2 k_{F}\left(k_{F}=\pi / 2\right.$ for the half-filled band case, the lattice constant has been set as unit). For the Grassmann variables we could introduce a spinor notation via 10

$$
\Phi_{k, s}=\left(\begin{array}{c}
\phi_{k+k_{F}, s} \\
\phi_{k-k_{F}, s}
\end{array}\right)
$$

which are right- and left-moving electrons, respectively, then we have the partition function as

$$
Z=\int \mathcal{D}\left[a^{*} a, b^{*} b, \Phi^{*} \Phi\right] e^{-S},
$$

and the corresponding action could be written as a sum of five terms

$$
S=S_{a}+S_{b}+S_{e}+S_{a-e}+S_{b-e}
$$

where the acoustic- and optical-phonon parts of the action are, respectively,

$$
\begin{aligned}
S_{a} & =\sum_{q, \Omega}\left[\frac{1}{2} K+\frac{1}{2} M \Omega^{2}\right] a_{q}(\Omega) a_{-q}(-\Omega), \\
S_{b} & =\sum_{q, \Omega}\left[\frac{1}{2} K+\frac{1}{2} M \Omega^{2}\right] b_{q}(\Omega) b_{-q}(-\Omega),
\end{aligned}
$$

the electronic part of the action is

$$
S_{e}=\sum_{k, \omega, s} \Phi_{k, s}^{*}(\omega)\left[(i \omega-\mu)+2 t_{0} \sin k \sigma_{3}\right] \Phi_{k, s}(\omega),
$$

and the acoustic and optical phonon-electron interaction parts are the following

$$
\begin{aligned}
& S_{a-e}=\frac{\alpha}{\sqrt{\beta L}} \sum_{q, \Omega} \sum_{k, \omega, s} a_{q}(\Omega) \Phi_{k, s}^{\dagger}(\omega) \Phi_{k-q, s}(\omega-\Omega), \\
& S_{b-e}=\frac{\alpha}{\sqrt{\beta L}} \sum_{q, \Omega} \sum_{k, \omega, s} b_{q}(\Omega) \Phi_{k, s}^{\dagger}(\omega) \sigma_{1} \Phi_{k-q, s}(\omega-\Omega),
\end{aligned}
$$

where $\sigma_{i}$ are Pauli matrices.

In the SSH model and the MX model, the optical phonon-electron interactions are dominant and the acoustic phonon-electron interactions can be ignored 10 However, as seen from the Eq. (8d) and (8e), the couplings between electrons and two branches of phonons are equivalent in the MC model. The fact implies we should take the both into our consideration. One can integrate out the electronic variables of the partition since the action is bilinear in the Grassmann fields, the resulting partition function is

$$
Z=\int \mathcal{D}\left[a^{*} a b^{*} b\right] e^{-S_{\mathrm{eff}}},
$$

and the effective action is

$$
\begin{aligned}
S_{\text {eff }}=\sum_{q, \Omega} & {\left[\frac{1}{2} K+\frac{1}{2} M \Omega^{2}\right]\left[a_{q}(\Omega) a_{-q}(-\Omega)+b_{q}(\Omega) b_{-q}(-\Omega)\right] } \\
& -N \ln \operatorname{det}(\mathcal{M}),
\end{aligned}
$$

where $N$ is the spin degree of freedom, det denotes the determinant of the matrix $\mathcal{M}$, which is defined by the actions $S_{e}+S_{a-e}+S_{b-e}=\sum_{s} \Phi_{s}^{\dagger} \mathcal{M} \Phi_{s}$ and has $k, \omega$, and the indices of the Pauli matrices as labels.

By the functional derivation of the effective action $S_{\text {eff }}$ with the phonon variables vanishing, we have the Peierls dimerization, i.e., the optical phonon condensation at zero momentum and zero frequency $\left\langle\alpha b_{q}(\Omega)\right\rangle=$ $\sqrt{\beta L} \Delta \delta_{q, 0} \delta_{\Omega, 0}$, where the phonon order parameter $\Delta$ describes the Peierls dimerization. Defining the noninteracting electronic Green's function $G_{0}(k, \omega)$ by

$$
G_{0}(k, \omega)=-\left(i \omega-\mu+2 t_{0} \sin k \sigma_{3}+\Delta \sigma_{1}\right)^{-1},
$$

and $b_{q}(\Omega)=\left\langle b_{q}(\Omega)\right\rangle+\tilde{b}_{q}(\Omega)$, we obtain the effective action by expanding the logarithm in order of $\tilde{b}$ and $a$ :

$$
S_{\text {eff }}=S_{\text {eff }}^{(0)}+\sum_{n=1}^{\infty} S_{\text {eff }}^{(n)} .
$$

$S_{\text {eff }}^{(0)}$ is the zeroth-order contribution in $\tilde{b}$ and $a$ and is given by

$S_{\mathrm{eff}}^{(0)}=N \beta L\left\{\frac{\Delta^{2}}{2 \lambda \pi t_{0}}-\int \frac{d k d \omega}{(2 \pi)^{2}} \ln \operatorname{det} G_{0}^{-1}(k, \omega)\right\}$,

in the thermodynamic limit, the dimensionless electronphonon coupling constant $\lambda$ is defined by $\lambda=$ $N \alpha^{2} / \pi K t_{0}$, the chemical potential $\mu=0$ at the half-filling case. The electronic spectrum $E_{k}=$ $\pm \sqrt{\left(2 t_{0} \sin k\right)^{2}+\Delta^{2}}$, the electronic gap is $2 \Delta$.

The mean field gap equation

$$
\Delta_{\mathrm{ad}}=\Lambda e^{-2 / \lambda}
$$

follows immediately from $\partial S_{\text {eff }}^{(0)} / \partial \Delta=0$, where $\Lambda$ is the integral cutoff. The mean-field result tell us that the ground state is dimerized for any electron-phonon interactions. To investigate the effects of quantum phonon fluctuations, we should include higher order contributions. The first-order term $S_{\text {eff }}^{(1)}$ vanishes since $S_{\text {eff }}^{(0)}$ is obtained from the saddle point approximation. The secondorder contribution to the effective phonon action is 


$$
\begin{aligned}
S_{\mathrm{eff}}^{(2)}= & \sum_{q, \Omega}\left\{a_{q}^{*}(\Omega) a_{q}(\Omega)\left[\frac{1}{2} M \Omega^{2}+\lambda K f(q, \Omega)\right]\right. \\
& \left.+\tilde{b}_{q}^{*}(\Omega) \tilde{b}_{q}(\Omega)\left[\frac{1}{2} M \Omega^{2}+\lambda K g(q, \Omega)\right]\right\},
\end{aligned}
$$

where the functions $f(q, \Omega)$ and $g(q, \Omega)$ are defined as follows

$$
\begin{array}{r}
f(q, \Omega)=\frac{1}{2 \lambda}+\frac{\pi t_{0}}{2} \int \frac{d k d \omega}{(2 \pi)^{2}} \operatorname{Tr}\left[G_{0}(k-q, \omega-\Omega)\right. \\
\left.G_{0}(k, \omega)\right], \\
g(q, \Omega)=\frac{1}{2 \lambda}+\frac{\pi t_{0}}{2} \int \frac{d k d \omega}{(2 \pi)^{2}} \operatorname{Tr}\left[G_{0}(k-q, \omega-\Omega)\right. \\
\left.\sigma_{1} G_{0}(k, \omega) \sigma_{1}\right] .
\end{array}
$$

By performing the integration in Eq. (9) over the fluctuations $\tilde{b}$ and $a$ to the second-order term, we have

$$
Z=e^{-\beta L \Gamma[\Delta]},
$$

where the free energy density $\Gamma[\Delta]$ is composed of two parts,

$$
\Gamma[\Delta]=\Gamma_{0}[\Delta]+\Gamma_{1}[\Delta]
$$

$\Gamma_{0}[\Delta]=S_{\text {eff }}^{(0)} / \beta L$ is the zeroth-order contribution, and the one-loop contribution $\Gamma_{1}[\Delta]$ is given by

$$
\begin{aligned}
\Gamma_{1}[\Delta]=\frac{1}{2} \int \frac{d q d \Omega}{(2 \pi)^{2}} & \left\{\ln \left[\frac{1}{2} M \Omega^{2}+\lambda K f(q, \Omega)\right]\right. \\
& \left.+\ln \left[\frac{1}{2} M \Omega^{2}+\lambda K g(q, \Omega)\right]\right\},
\end{aligned}
$$

in the zero-temperature and thermodynamic limit. The equation for the phonon order parameter $\Delta$ is determined by $\partial \Gamma[\Delta] / \partial \Delta=0$. Within the one-loop approximation, we have

$$
\begin{aligned}
1=2 \pi \lambda t \int \frac{d q d \Omega}{(2 \pi)^{2}} & \left\{\frac{1}{\Omega^{2}+E_{\mathrm{q}}^{2}}\right. \\
& \left.-\frac{1}{N} \cdot \frac{R_{f}^{-1}(q, \Omega)+R_{g}^{-1}(q, \Omega)}{4 \Delta}\right\},
\end{aligned}
$$

where

$$
R_{u}(q, \Omega) \equiv \frac{\Omega^{2}}{\Omega_{0}^{2} u^{\prime}(q, \Omega)}+\frac{u(q, \Omega)}{u^{\prime}(q, \Omega)},
$$

with both $u=f$ and $g$, the renormalized phonon frequency $\Omega_{0}^{2}=2 \lambda \omega_{Q}^{2}\left(\omega_{Q} \equiv \sqrt{K / M}\right)$. In the limit of weak electron-phonon interaction, only electrons near the Fermi surfaces be important, that is, we can take small momentum approximation in the calculation of the functions $f(q, \Omega)$ and $g(q, \Omega)$, which gives $f(q, \Omega)=g(q, \Omega)$. This result implies the equivalence of the contributions from the optical and acoustic phonons in quantum fluctuations in the MC model. The derivation of the function $u(q, \Omega)$ with the phonon order parameter $\Delta$ can be calculated straightforwardly, it gives $u^{\prime}(q, \Omega) / u(q, \Omega)=$ $4 \Delta /\left(\Omega^{2}+4 t_{0}^{2} q^{2}+4 \Delta^{2}\right)$. Then we have

$$
R_{u}(q, \Omega)=\left(d^{2} \Omega^{2}+4 t_{0}^{2} q^{2}+4 \Delta^{2}\right) / 4 \Delta,
$$

with the abbreviation $d^{2}=1+4 \Delta^{2} / \Omega_{0}^{2}$, the higher-order terms have been neglected as usual (in a renormalizationgroup study). The equation (20) can be compared with that in the SSH or MX model where only one $R_{u}$ appears since the acoustic phonon-electron interaction can be ignored, so that the spin- $\frac{1}{2}(N=2)$ MC model is equivalent to the spinless $(N=1) \mathrm{SSH}$ or MX model in this sense.

Now we obtain the phonon order parameter

$$
\Delta=\Lambda \exp \left[-2\left(1-\frac{\lambda \ln 2}{N d}\right) / \lambda\left(1-\frac{2}{N d}\right)\right],
$$

by performing the integral of Eq. (20). It can be seen that in the adiabatic limit $M \rightarrow \infty$, the quantum fluctuation is completely suppressed and the above equation returns to the mean-field one (14).

The results obtained from Eq. (23) are discussed as follows. (1) For the spinless electron systems, the ground state is undimerized for a weak electron-phonon coupling whereas it is dimerized for a strong electron-phonon coupling. The numerical results are in gopd agreement with that of the Monte Carlo simulations 3 (2) For the spin- $\frac{1}{2}$ electron systems, the ground state is undimerized in the limit of $M=0$; it is dimerized for the system with any nonzero mass in the limit of strong electronphonon interactions. This resulf agrees with that of the renormalization-group analysis. 3 (3) A typical curve of our numerical results for the $N=2$ case is given in Fig. 1 . To compare the data we obtain with that of the Monte Carlo calculation, we used the phonon-staggered order parameter $m_{p}$, which is related to $\Delta$ through $m_{p}=\Delta / \alpha$. It is found that the Peierls dimerization is destroyed in weak electron-phonon coupling regime. This is consistent with the spinless SSH or MX model since they are equivalent. The data we obtained are quite close to that of Monte Carlo simulation, 3 in which a jump in $m_{p}$ is seen at a critical $\alpha$. We believe that this is a signature of the transition discussed in the present work, although an opsite conclusion was reached at by the original authors. This result also implies that the break of the continuous symmetry in spin space does not necessarily produce dimerization although the continuous symmetry does prevent the existence of long- range dimerization order in the model. 3 Finally, we mention the works by a variational approach, 6 , which can not give out a transition since it underestimates the quamtun fluctuations.

In summary, the effects of quantum phonon fluctuations on Peierls dimerization in the molecular crystal model are reexamined by a functional integral approach. The calculation is performed by first integrating out the electronic variables of the partition function and then expanding the effective action to the quadratic terms in 
the phonon variables. It is found that the Peierls dimerization is destroyed by the quantum phonon fluctuations for the spin- $\frac{1}{2}$ electron systems. This result favors earlier suggestion by Little11 on the possible high- temperature superconductivity in this kind of systems.

The authors are grateful to the Shanghai Qi-MingXing Plan for Young Scientists, the State Education Commission of China, and the National Nature Science Foundation of China for their support.

* Electronic address: cqwu@fudan.ihep.ac.cn

${ }^{1}$ R.E. Peierls, Quantum Theory of Solids (Oxford University Press, London, 1955).

${ }^{2}$ E. Fradkin and J.E. Hirsch, Phys. Rev. B 27, 1680 (1983).

${ }^{3}$ J.E. Hirsch and E. Fradkin, Phys. Rev. B 27, 4302 (1983).

${ }^{4}$ W.P. Su, J. Schrieffer, and A.J. Heeger, Phys. Rev. B 22, 2099 (1980).

${ }^{5}$ T. Holstein, Ann. Phys. 8, 325 (1959).

${ }^{6}$ H. Zheng, D. Feinberg, and M. Avignon, Phys. Rev. B 39, 9405 (1989).

${ }^{7}$ H. Zheng, Phys. Rev. B 50, 6717 (1994).

${ }^{8}$ D. Schmeltzer, R. Zeyher, and W. Hanke, Phys. Rev. B 33, 5141 (1986).

${ }^{9}$ Q.F. Huang, C.Q. Wu, and X. Sun, Phys. Rev. B 52, Aug. 15th (1995).

${ }^{10}$ Z.B. Su, Y.X. Wang, and L. Yu, Commun. Theor. Phys. 6, 313 (1986).

${ }^{11}$ W.A. Little, Phys. Rev. 134, A1416 (1964).

FIG. 1. The dependence of the phonon-staggered order parameter $m_{p}$ on the electron-phonon coupling $\alpha$ for $\omega_{Q}=1.1$, $K=0.25$, and $t_{0}=1$. The dashed line is the mean-field result and the data with error bar are the Monte Carlo results. 